\title{
Evolução na utilização e nos gastos de uma operadora de saúde
}

\author{
Evolution in the use and the expenditures of a healthcare provider
}

André Luís de Castro Moura Duarte ${ }^{1}$

Felippe de Medeiros Oliveira ${ }^{1}$

Anderson de Andrade Santos ${ }^{1}$

Bento Fortunato Cardoso dos Santos ${ }^{1}$

${ }^{1}$ Insper Instituto de Ensino e Pesquisa. Rua Quatá 300, Vila Olímpia. 04546042 São Paulo SP Brasil. andrelcmd@insper.edu.br
Abstract Brazil's aging population and the rising number of people reliant upon the country's supplementary healthcare system have elicited the concern of public and private managers regarding the increase in healthcare costs. In this paper, the costs per gender, per type of medical expenses and per age group of a major Brazilian self-managed healthcare provider between 2007 and 2013 were analyzed. This healthcare provider is of interest because, besides portraying a single condition of revenue growth restricted to the existing contributors, it also replicates the demographic profile expected for Brazil in 2050, when approximately one-third of its population will be over 60 years of age. The analyses confirm the current literature as they show an increase in healthcare plan usage by the elderly and the difference between admission rates by gender. They also reveal an increase in average length of stay in hospital and the increase in medical costs far above inflation, especially for materials and medicines. It is hoped that this study will help scholars and others interested in comparisons of medical expense trends, especially by age and sex, and that it encourages further collaboration on the sustainability of health insurance providers in Brazil.

Key words Hospital cost evolution, Aging population, Gender, Self-managed provider, Health services
Resumo O envelhecimento da população e o crescimento no número de pessoas expostas aos cuidados do sistema de saúde suplementar no Brasil aumentam a preocupação dos gestores públicos $e$ privados com o crescimento dos custos da área de saúde. Neste trabalho foram analisados os custos por gênero, por tipo de gasto médico e por faixa etária de uma operadora de autogestão brasileira no periodo entre 2007 a 2013. Tal operadora é de grande interesse pois além de retratar uma condição única de restrição de crescimento de receita, também replica o perfil demográfico esperado para o Brasil no ano de 2050, quando aproximadamente um terço da sua população estará acima dos 60 anos. As análises corroboram a literatura vigente uma vez que demonstram um aumento na utilização do plano pelos mais idosos, e apontam para uma diferença nas taxas de internação entre os gêneros. O estudo também mostra um expressivo aumento no tempo médio de permanência nos hospitais e mostra crescimento dos gastos médicos muito acima da inflação, com destaque para materiais e medicamentos. No geral, esperamos que o presente estudo auxilie estudiosos e interessados em futuras comparações dos gastos médicos por idade e gênero e que colabore na sustentabilidade das operadoras de saúde no Brasil.

Palavras-chave Evolução dos custos hospitalares, Envelhecimento populacional, Gênero, Operadora de autogestão, Serviços de saúde 


\section{Introdução}

O sistema de saúde no Brasil pode ser dividido em duas grandes frentes: o Sistema Único de Saúde (SUS) e o sistema de saúde privado ${ }^{1,2}$. O SUS trata-se do serviço público oferecido de maneira universal a todo cidadão brasileiro; já o sistema de saúde privado é prestado por meio da contratação direta de serviços junto a prestadores particulares, entre eles hospitais, clínicas e operadoras de planos de saúde, que juntas formam o Sistema de Saúde Suplementar ${ }^{3}$.

Nos últimos anos, o sistema de saúde suplementar brasileiro vem apresentando crescimento expressivo ${ }^{4}$. Segundo dados da Agência Nacional de planos de Saúde Suplementar (ANS) o número total de beneficiários de planos médico-hospitalares atingiu 50,8 milhões em dezembro de 2014, apresentando crescimento significativo nos últimos anos ${ }^{5,6}$. A mesma fonte aponta para um crescimento no número de beneficiários de $13,1 \%$ entre os anos de 2010 a 2014, sendo que no mesmo período as receitas subiram mais de $60 \%$. Estes números retratam a importância crescente do setor de saúde suplementar para a economia brasileira ${ }^{7}$. Mesmo com o aumento de beneficiários, vale destacar que a mesma ANS aponta redução no número de operadoras no segmento médico-hospitalar com beneficiários, sendo 1044 em 2010 e 873 em 20145.

Apesar do crescimento de beneficiários da saúde suplementar no Brasil, uma das maiores preocupações do setor está relacionada ao aumento dos custos da saúde no país. São várias as justificativas apontadas pelas empresas do setor para o aumento destes custos, entre elas o envelhecimento da população ${ }^{8}$, o aumento da frequência de utilização dos planos de saúde ${ }^{9}$ e o aumento de preços de medicamentos, mão de obra e equipamentos ${ }^{10,11}$. Este incremento nos custos é verificado pelo aumento do Índice de Preços ao Consumidor do Município de São Paulo, em 2014. Enquanto o IPC Geral foi de $5,20 \%$, o IPC Saúde foi de $7,02 \%$. A diferença apresentada fica ainda maior quando consideramos o período entre 2005 e 2014, quando o IPC Geral acumulado foi de $59,18 \%$ e o IPC Saúde chegou a $92,45 \%{ }^{12}$.

Dentro deste contexto e com a intenção de contribuir para um melhor entendimento dos gastos da saúde suplementar no Brasil, este estudo tem como objetivo principal avaliar a evolução dos gastos e da utilização dos serviços de saúde em uma operadora de autogestão brasileira por gênero (masculino e feminino $)^{13}$, por tipo de gasto médico e por faixa etária (mais velhos - acima de 60 anos e mais novos - abaixo de 60 anos) ${ }^{14}$.
Para alcançar este objetivo foi analisada, durante o período de 2007 a 2013, a evolução dos gastos de uma operadora de saúde do tipo autogestão, com cerca de 90 mil beneficiários, nas categorias ambulatorial, internação e domiciliar, sendo a primeira subdividida em consultas, exames, procedimentos clínicos e procedimentos cirúrgicos. Já os gastos com internações foram subdivididos em diárias, exames, materiais, medicamentos, terapias, taxas, honorários e outras despesas.

A escolha da operadora para este estudo se deve a alguns fatores que a tornam única, tais como: o reduzido número de beneficiários ingressantes, uma vez que a entrada destes está limitada (102 mil em 2008 para 81 mil em 2014); o envelhecimento acelerado dos mesmos (44 anos em 2008 para 47,6 anos em 2012); e a forte concentração deles na cidade de São Paulo e na grande São Paulo (aproximadamente 44\%). Fatores estes que potencializam a elevação dos custos, algo presente ou esperado em muitas operadoras de saúde brasileiras.

\section{Revisão da literatura}

A cadeia de valor da área de saúde suplementar no Brasil é descrita segundo o Guia da Saúde do Instituto de Estudos de Saúde Suplementar ${ }^{15}$ como um sistema que envolve os prestadores de serviço, como hospitais, clínicas, laboratórios e centros de diagnóstico; profissionais da saúde, como médicos, fisioterapeutas, psicólogos, nutricionistas; a indústria farmacêutica e de equipamentos da saúde; as operadoras de planos de saúde; as farmácias e as distribuidoras de medicamentos e as agências governamentais reguladoras Agência Nacional de Vigilância Sanitária (Anvisa) e ANS. Essa cadeia de valor forma uma trama de relacionamentos complexa de prestação de serviços a empresas e ao cliente final (Figura 1).

Para a Organização Mundial da Saúde ${ }^{16}$, um sistema de saúde é a soma de todas as organizações, instituições e fundos que têm o propósito primário de melhorar a saúde. São necessários equipes, recursos, informações, fornecedores, transporte e comunicações, além de diretrizes de gestão integradas.

Para que estes serviços apresentem preços justos, é fundamental que os gastos destas organizações sejam bem gerenciados. Neste sentido, alguns estudos mostram grande preocupação com o aumento dos custos da área de saúde no Brasi ${ }^{7}, 17,18$. Uma das principais questões usadas para explicar o aumento dos custos da saúde de 
Orgãos de Regulação (ANS)

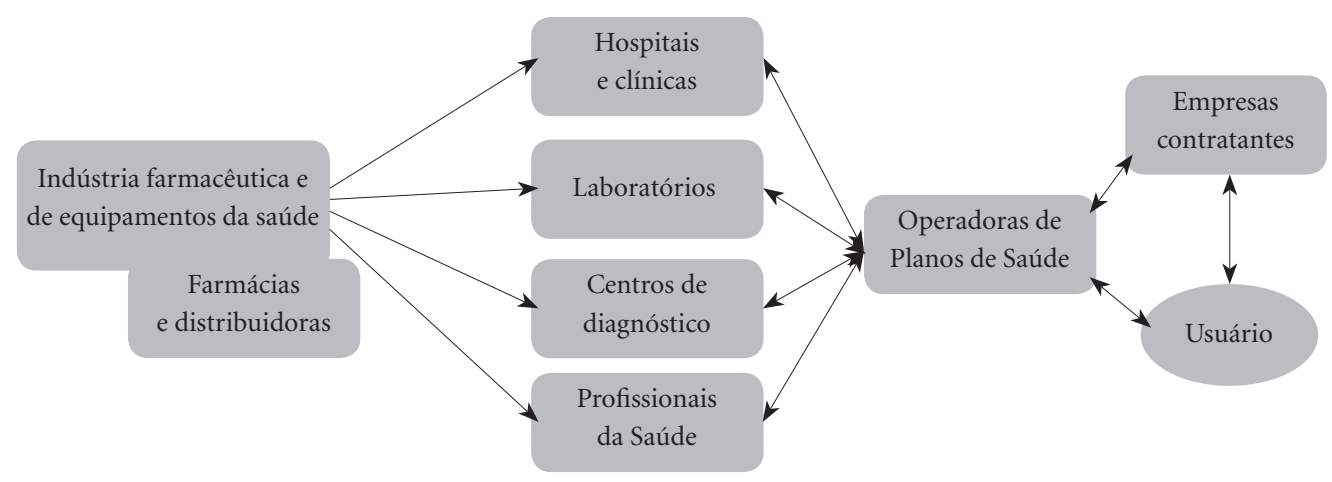

Atividades periféricas como academias, clínicas e profissionais de terapias alternativas

Figura 1. Representação do Sistema de Saúde Privado ${ }^{19}$.

um país é o envelhecimento da população ${ }^{20}$. A população brasileira apresenta desafios para a cadeia de valor da área de saúde, pois, conforme o relatório dos indicadores do IBGE, as pessoas com mais de 60 anos de idade representavam 13\% da população em $2013^{21}$. Além disso, há estimativas de que esse contingente possa triplicar até $2050^{22}$. Para buscar uma melhor compreensão do impacto desse processo, faz-se necessário abordar algumas características dentro desse público.

O primeiro deles trata da diferença no padrão de gastos entre os gêneros. A literatura aponta que as mulheres, em geral, tendem a utilizar mais serviços de saúde do que os homens ${ }^{23}$. Além disto, a quantidade de mulheres é superior à de homens em todas as idades na saúde suplementar. Isto fica evidente entre os idosos com acesso a planos de saúde ${ }^{23}$ : por exemplo, para cada 100 homens acima dos 60 anos de idade, existem 143 mulheres e para os idosos acima de 80 anos, essa diferença quase dobra ${ }^{22}$. Em outro estudo ${ }^{24}$, foram analisados os indicadores de gastos médicos e constatou-se evidências de que as mulheres têm o gasto 1,29 vezes superior aos homens em consultas e 1,39 vezes em exames, embora os gastos masculinos em internações tenham sido 1,12 vezes superiores aos das mulheres. De acordo com os resultados, os gastos com internação são 67 ve- zes superiores aos gastos médios com consultas. Portanto, a relevância dos gastos com internações é inquestionável para operadoras de saúde ${ }^{24}$.

O envelhecimento populacional também afeta os gastos. Estima-se, por exemplo, que em 2030 o gasto público com assistência hospitalar e ambulatorial no SUS poderá aumentar 43,9\% em relação a $2010^{22}$. Dados atualizados do relatório "Envelhecimento Populacional e os Desafios para o Sistema de Saúde Brasileiro” apontam que o gasto relativo ao grupo de idosos aumentará significativamente. Destaca-se a contribuição dos gastos totais para o grupo com mais de 60 anos: esse grupo representou $28 \%$ do gasto total em 2010, e estima-se que esse percentual represente $59 \%$ em $2050^{9}$.

Outro ponto de atenção para as operadoras encontra-se na ineficiência da gestão dos custos ${ }^{17}$, ocasionando aumentos progressivos. Estudo realizado pelo IESS (Instituto de Estudos de Saúde Suplementar) no Brasil mostra que há uma tendência de gastos crescentes ${ }^{10}$. Os gastos com internação cresceram, entre 2005 e 2010, mais de $54 \%$, sendo que materiais e medicamentos representaram a maior parcela do gasto e do crescimento. Ainda segundo os próprios autores, estes resultados suscitam discussões relacionadas ao impacto do uso de novas tecnologias, relaciona- 
das a materiais e medicamentos, nos custos assistenciais das operadoras de saúde, corroborando com estudos internacionais que apontam o avanço na tecnologia como um dos principais responsáveis pelo aumento dos gastos com saúde ${ }^{25}$.

Diante de todo este cenário, torna-se essencial o aprimoramento da gestão hospitalar e o pleno controle da sua administração de custos e eficiência na prestação de serviços. Com a aceleração do aumento de seus gastos, constatou-se que muitas operadoras, em especial as cooperativas, estão partindo para a integração vertical de suas operações. Em pesquisa, foi concluído que há um grupo significativo de operadoras que está internalizando suas atividades de prestação de serviços hospitalares, exames e aquisição de insu$\operatorname{mos}^{26}$. Esses resultados sugerem esforços estratégicos das operadoras para direcionar o aumento da utilização da rede própria.

\section{A empresa pesquisada e o método de análise}

Esta pesquisa se caracteriza por seu caráter não experimental e descritivo ${ }^{27}$, uma vez que tem como finalidade observar, registrar e analisar o fenômeno do aumento dos gastos do Sistema de Saúde Suplementar Brasileiro.

Para este objetivo usou-se o método do estudo de caso extremo ${ }^{28}$ com uma operadora de plano de saúde que passa por uma forte redução no número de beneficiários, pelo envelhecimento acelerado dos mesmos e que, além disto, apresenta uma forte concentração de usuários na grande São Paulo. Todas estas características potencializam o fenômeno que se deseja estudar: o aumento dos gastos de uma operadora de saúde. A vantagem de utilização de casos extremos está em fornecer uma ideia dos limites dentro dos quais as variáveis de estudo podem oscilar ${ }^{28}$.

Sendo assim, a empresa objeto deste estudo é uma operadora de Plano de Saúde que atua sob a modalidade de autogestão, criada na década de 1960 para atender os funcionários de uma grande empresa pública privatizada no início do século XXI. Oferece a seus beneficiários diversos tipos de plano de saúde com cobertura a consultas, exames, tratamentos clínicos e cirúrgicos, internações hospitalares, saúde mental e assistência odontológica. A maior parte de seus beneficiários, aproximadamente 90\%, concentra-se no estado de São Paulo, apesar da presença em outros estados em regime de reciprocidade com outros planos de saúde. Atualmente, sua assistência é extensível até o $3^{\circ}$ grau de parentesco por consanguinidade ou $2^{\circ}$ grau por afinidade, do associado e ex-associado. A empresa conta com uma rede de 12.300 credenciados, entre hospitais, clínicas, médicos, laboratórios de exames e outros.

As análises foram desenvolvidas a partir dos relatórios de gastos da própria empresa. Foram disponibilizadas para análise as informações relativas aos anos de 2007 a 2013. Vale ressaltar que o volume de contratos de coparticipação é mínimo, logo, a remuneração dos hospitais, clínicas e médicos é de total responsabilidade da operadora.

Para a análise dos dados disponibilizados pela empresa foram utilizadas técnicas de estatística básica descritiva. Algumas faixas etárias, consideradas outliers, seja pela pequena quantidade de pessoas ou pelos altos custos do período, foram retiradas da análise, de forma a conferir a menor distorção possível. As análises foram divididas nas seguintes categorias: ambulatorial, internação e domiciliar. As duas primeiras se mostraram mais relevantes e desta forma foram subdivididas. Os gastos ambulatoriais foram subdivididos em consultas, exames, procedimentos clínicos e procedimentos cirúrgicos. Já os gastos com internações foram subdivididos em diárias, exames, materiais, medicamentos, terapias, taxas, honorários e outras despesas.

\section{Análise dos dados e resultados}

Inicia-se esta análise com alguns dados do perfil e evolução etária dos beneficiários da operadora de saúde estudada. O número médio de expostos caiu 17\% entre 2007 e 2013 . Esta queda, conforme abordado anteriormente, pode ser explicada pelo envelhecimento da população do plano e pela quase inexistente entrada de novos beneficiários. Houve uma queda de $30 \%$ no número de beneficiários abaixo de 60 anos e um aumento de 26\% naqueles acima dessa idade. Como observado no Gráfico 1, se em 2007 a quantidade de beneficiários abaixo de 60 anos representava 76\% da população, em 2013 este número caiu para $64 \%$. Outra observação importante a ser feita é que enquanto o número de beneficiários do sexo masculino acima de 60 anos cresceu de 10\% para $16 \%$, no sexo feminino, nessa faixa etária, subiu de $13 \%$ para $20 \%$ do total. Ou seja, o número de mulheres mais velhas cresceu mais rapidamente do que o número de homens mais velhos no período, corroborando os estudos realizados por Carneiro et al. ${ }^{20}$.

No total, a proporção do número de mulheres e homens não foi alterada de forma significativa durante o período de 2007 a 2013, sendo aproximadamente $55 \%$ de beneficiários do sexo feminino e $45 \%$ do masculino. 
Após apresentação do perfil e da evolução etária dos beneficiários da operadora de saúde, será apresentada a evolução da utilização e dos gastos dos beneficiários mais velhos (acima de 60 anos) comparativamente com os mais novos (abaixo de 60 anos) para ambos os sexos.

\section{Análise da Evolução da Utilização dos Serviços de Saúde}

A seguinte análise antecipa um passo importante das tendências nos gastos da operadora em questão. As evoluções na utilização dos serviços de saúde impactam diretamente, assim como o gênero e a idade - como já apontados em nossa revisão de literatura anterior - no custo da operadora. Portanto, serão analisados o perfil de uso da operadora por seus beneficiários e a relação deste com a taxa de utilização dos serviços médicos.

Observou-se, no período referente, que a taxa média de utilização da operadora aumentou de $41 \%$ para $49 \%$ (Tabela 1). O crescimento na taxa de utilização está relacionado positivamente ao aumento da frequência média de utilização de exames ambulatoriais por beneficiário, passando de 14,7 em 2007 para 27,02 exames/beneficiário em 2013. A mesma relação foi encontrada com o aumento de consultas por beneficiário, que saltaram de 5,12 em 2007 para 6,25 consultas/beneficiário em 2013. Ambos relevantes na sequência de custos incorridos ao plano de saúde.

Tal evolução na utilização - e consequente impacto nos custos - também foi constatado por faixa etária. Tanto os beneficiários mais novos quanto os mais velhos aumentaram a utilização do plano, sendo encontrado neste último um ritmo ainda mais acelerado que o primeiro, saltando de 49\% em 2007 para 61\% em 2013 (Tabela 2). Na avaliação por gênero, ambos aumentaram a utilização do plano de saúde, sendo as mulheres, em média, as maiores utilizadoras, em ambas faixas etárias. Primeiramente, deve-se destacar a diferença na utilização do plano entre os mais jovens, sendo mantida a diferença de aproximadamente 11 pontos percentuais dos anos demonstrados (vide taxa de utilização na Tabela 3, na qual $46 \%$ das mulheres utilizaram o plano frente aos 35\% dos homens no ano de 2013). Observou-se, entretanto, que os homens acima de 60 anos também aumentaram significativamente a taxa de utilização no período analisado (de $48 \%$ para $62 \%$ ) e praticamente igualaram o nível de

Tabela 1. Evolução das taxas de utilização geral, exames por expostos e consultas por beneficiários.

\begin{tabular}{cccc}
\hline Ano & $\begin{array}{c}\text { Taxa de } \\
\text { utilização }\end{array}$ & $\begin{array}{c}\text { Exames por } \\
\text { exposto }\end{array}$ & $\begin{array}{c}\text { Consultas } \\
\text { Beneficiário }\end{array}$ \\
\hline 2007 & $41 \%$ & 14,7 & 5,12 \\
2008 & $46 \%$ & 20,6 & 6,16 \\
2009 & $46 \%$ & 20,1 & 5,67 \\
2010 & $47 \%$ & 21,1 & 5,51 \\
2011 & $48 \%$ & 23,5 & 5,85 \\
2012 & $48 \%$ & 25,2 & 5,93 \\
2013 & $49 \%$ & 27,0 & 6,25 \\
\hline
\end{tabular}

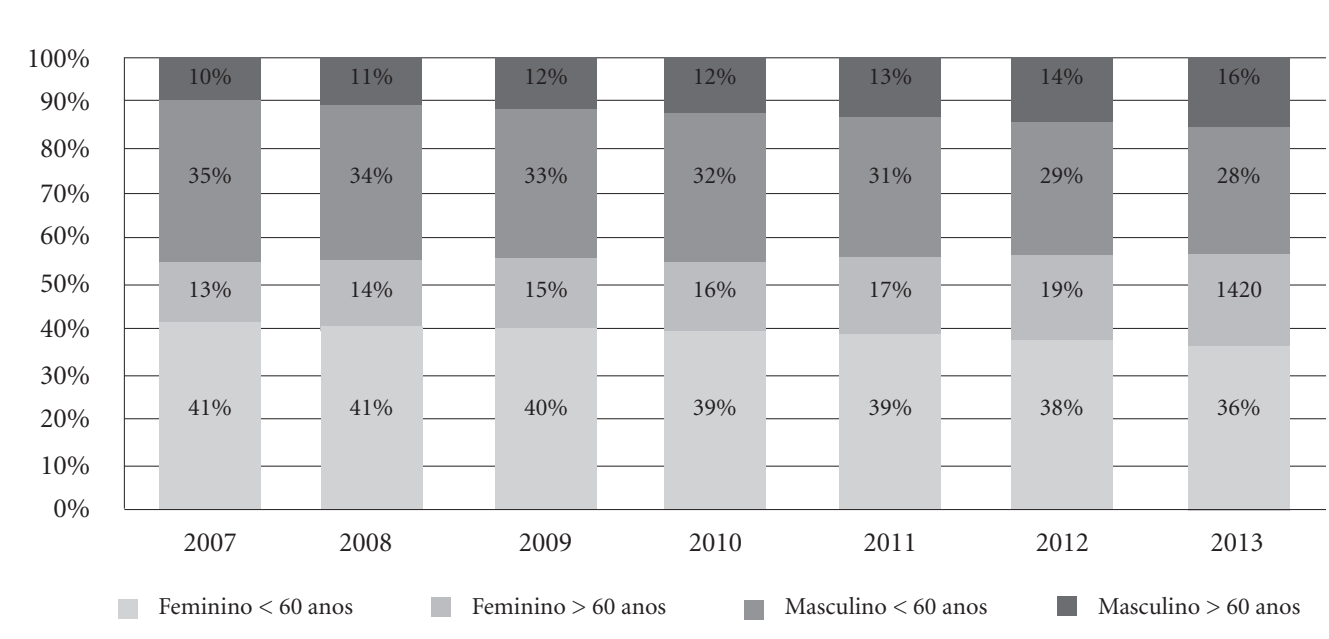

Gráfico 1. Evolução do perfil (sexo e faixa etária) dos beneficiários da Operadora de Saúde no período de 2007 a 2013. 
utilização das mulheres na faixa acima de 60 anos (Tabela 3).

O desdobramento das análises por idade, gênero e tipo de gasto apresentados na Tabela 3 apontam para a tendência de aumento na utilização do plano nas idades mais avançadas. Em 2013, por exemplo, a taxa de exames por beneficiário para a população mais velha foi de 37,3 exames por indivíduos, enquanto que para a população mais nova foi de 21,3. O mesmo fenômeno também é verificado no número de consultas por beneficiários: em 2013 a operadora tinha 7,5 consultas por indivíduo acima de 60 anos, contra 5,5 abaixo dessa idade.

Ainda na Tabela 3, pôde-se observar a diferença de comportamento entre os sexos - relevante para as extrapolações do comportamento dos gastos da operadora de saúde em questão sendo as mulheres, em média, as maiores utilizadoras de exames e consultas. Enquanto as mu-

Tabela 2. Evolução das taxas de internação e utilização para beneficiários acima e abaixo dos 60 anos.

\begin{tabular}{ccccc}
\hline \multirow{2}{*}{ Ano } & \multicolumn{2}{c}{ Taxa de Internação } & \multicolumn{2}{c}{ Taxa de Utilização } \\
\cline { 2 - 5 } & $<\mathbf{6 0}$ anos & $>\mathbf{6 0}$ anos & $<\mathbf{6 0}$ anos & $>\mathbf{6 0}$ anos \\
\hline 2007 & $10 \%$ & $35 \%$ & $35 \%$ & $49 \%$ \\
2008 & $12 \%$ & $47 \%$ & $41 \%$ & $53 \%$ \\
2009 & $9 \%$ & $42 \%$ & $39 \%$ & $55 \%$ \\
2010 & $10 \%$ & $41 \%$ & $39 \%$ & $58 \%$ \\
2011 & $10 \%$ & $39 \%$ & $40 \%$ & $59 \%$ \\
2012 & $10 \%$ & $41 \%$ & $40 \%$ & $60 \%$ \\
2013 & $12 \%$ & $35 \%$ & $41 \%$ & $61 \%$ \\
\hline
\end{tabular}

lheres mais velhas fizeram, em 2013, uma média de 39,93 exames por beneficiária, os homens da mesma faixa etária fizeram 34,03 . No caso das consultas, as mulheres mais velhas fizeram na média 8,20 e os homens 6,66.

Com relação à taxa média de internação, outro fator de ponderação em futuras tendências de gastos com saúde, observou-se (Tabela 2) que apesar de ser bem maior para os maiores de 60 anos (35\% para os mais velhos e $12 \%$, para os mais novos em 2013) não apresentou evolução significativa para o período analisado (na média subiu de $20 \%$ para 22\%). Já a Tabela 3 mostrou que enquanto as mulheres tiveram taxas de internação diminuindo ao longo do tempo, os homens apresentaram movimento inverso. No caso masculino, a taxa de internação para os mais novos subiu de $9 \%$ para $14 \%$, enquanto que para os mais velhos aumentou quase 10 pontos percentuais, de $35 \%$ para $44 \%$.

Embora a taxa de internação geral tenha se mantido praticamente estável, observou-se que a permanência por internação subiu consideravelmente. A média geral subiu de 4,91 para 6,51 dias por internação. Para os beneficiários abaixo de 60 anos esta taxa foi de 3,63 dias para 4,54 dias e para os beneficiários acima de 60 anos o aumento foi ainda maior: de 6,41 dias para 8,01 dias.

\section{Análise da Evolução dos Gastos da Operadora de Saúde}

Para o período de 2007 a 2013 o IPC Geral Acumulado foi de 40,33\% e o IPC Saúde foi ainda maior, 54,26\%. É importante ter estes núme-

Tabela 3. Evolução das taxas de internação e utilização para beneficiários acima e abaixo dos 60 anos dos sexos masculino e feminino.

\begin{tabular}{|c|c|c|c|c|c|c|}
\hline Ano & Sexo & Faixa Etária & $\begin{array}{c}\text { Taxa de } \\
\text { Internação }\end{array}$ & $\begin{array}{c}\text { Taxa de } \\
\text { Utilização }\end{array}$ & Exames/Expostos & Consulta/Expostos \\
\hline \multirow[t]{6}{*}{2007} & Feminino & $<60$ anos & $11 \%$ & $41 \%$ & 14,53 & 5,51 \\
\hline & & $>60$ anos & $35 \%$ & $50 \%$ & 25,27 & 7,33 \\
\hline & Masculino & $<60$ anos & $9 \%$ & $29 \%$ & 8,72 & 3,56 \\
\hline & & $>60$ anos & $35 \%$ & $48 \%$ & 21,76 & 5,72 \\
\hline & Ambos & $<60$ anos & $10 \%$ & $35 \%$ & 11,86 & 4,61 \\
\hline & & $>60$ anos & $35 \%$ & $49 \%$ & 23,76 & 6,64 \\
\hline \multirow[t]{6}{*}{2013} & Feminino & $<60$ anos & $10 \%$ & $46 \%$ & 25,82 & 6,57 \\
\hline & & $>60$ anos & $28 \%$ & $60 \%$ & 39,93 & 8,20 \\
\hline & Masculino & $<60$ anos & $14 \%$ & $35 \%$ & 15,49 & 4,25 \\
\hline & & $>60$ anos & $44 \%$ & $62 \%$ & 34,03 & 6,66 \\
\hline & Ambos & $<60$ anos & $12 \%$ & $41 \%$ & 21,30 & 5,55 \\
\hline & & $>60$ anos & $35 \%$ & $61 \%$ & 37,35 & 7,52 \\
\hline
\end{tabular}


ros em mente para que se possa comparar com os números encontrados na operadora estudada.

No mesmo período os gastos médicos gerais da operadora de saúde, em função do envelhecimento de seus beneficiários, tiveram um aumento de $119 \%$, bem acima da inflação no período. O aumento foi de $161 \%$ para o grupo de beneficiários acima de 60 anos. Este crescimento ocorreu basicamente em função do aumento na quantidade de pessoas desta faixa etária.

No entanto, quando é analisada a taxa de crescimento dos gastos per capita (por beneficiário), observa-se que enquanto o aumento de gastos foi de $107 \%$ para a população mais velha, para a mais nova foi de $147 \%$. Ainda assim os gastos por beneficiário mais velho são 3,2 vezes maiores quando comparados aos mais novos.

Dentre as categorias de gastos, os domiciliares foram os que mais cresceram (206\%), seguidos por aqueles com internação (137\%) e pelos ambulatoriais (91\%). Todos cresceram bem acima da inflação, mas chama atenção o aumento dos gastos com internação, que já eram os mais altos.

O Gráfico 2 mostra que os gastos com internação representavam 49,6\% do total em 2007 e em 2013 passaram a representar $53,5 \%$. Os gastos ambulatoriais, ao contrário, tiveram sua participação no total reduzida de $45,7 \%$, em 2007 , para 39,9\%, em 2013.

Dentro das categorias de gastos ambulatoriais, o mais significativo em 2013 foi com exames, representando $61 \%$, seguido por consultas
(26\%), procedimentos clínicos $(8 \%)$ e procedimentos cirúrgicos (5\%).

Focando na análise dos gastos com internações, observou-se no período avaliado um aumento médio de $141 \%$ por internação, porém quando são analisados os gastos diários de internação este crescimento é inferior, de $81 \%$, ainda assim bem acima da inflação do período (IPC Saúde: 54,26\%). A explicação para isto está no aumento da permanência média das internações já apontadas na seção anterior. Vale notar que, embora os gastos por internação para os beneficiários mais velhos sejam na média o dobro daqueles por internação para os mais novos, quando se observa estes custos por diária de internação esta diferença praticamente desaparece. Ou seja, o beneficiário mais velho não paga a mais por diária de internação, na verdade ele fica mais tempo internado e, por isto, os seus gastos com internação são maiores. Deve-se destacar ainda que, no período analisado, não houve diferença significativa na inflação dos gastos com internação entre a população mais velha (acima de 60) e a mais nova (abaixo de 60).

Conforme se observa no Gráfico 3, o gasto com materiais foi o que mais cresceu em relação aos demais custos da internação, pois em 2007 representava 30\% e em 2013 passou para 41\%. A inflação do gasto com materiais, no período pesquisado, também foi a mais alta (Tabela 3), enquanto que em relação à diária de internação subiu $146 \%$. O segundo maior gasto, que foi o

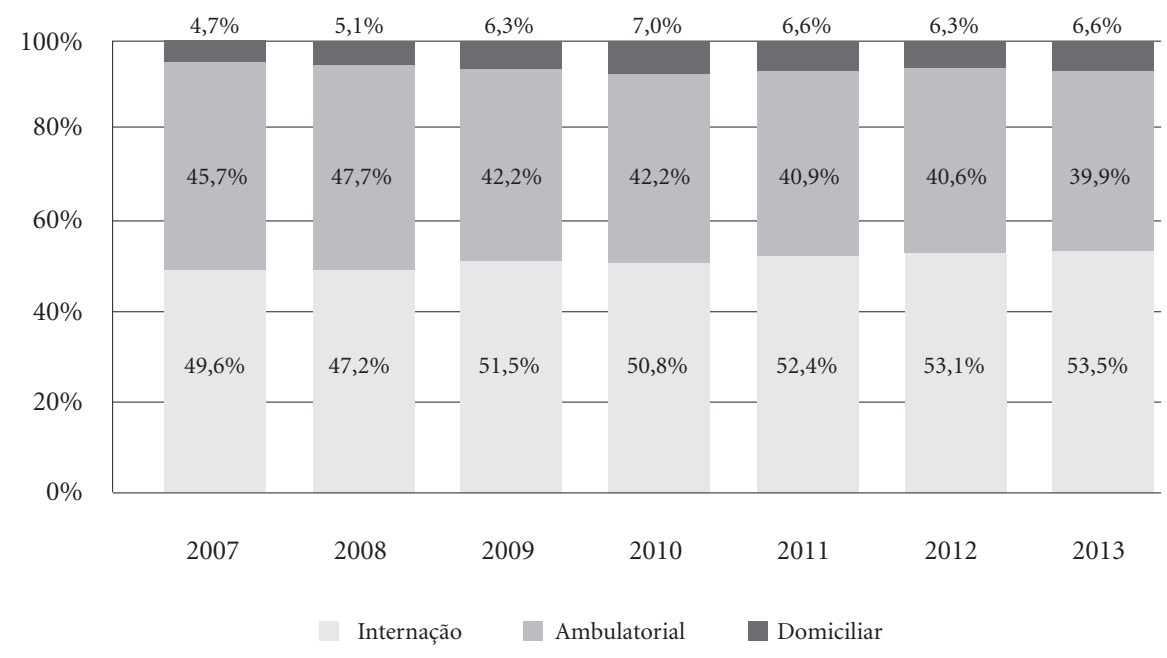

Gráfico 2. Evolução da representatividade dos gastos médicos totais com internação, ambulatorial e domiciliar. 
de medicamentos, subiu $67 \%$ por diária de internação.

Este dado corrobora a pesquisa de Leite $^{29}$, a qual afirma que o aumento dos custos relacionados a materiais tem sido um desafio para o setor de saúde, tanto pública quanto suplementar. Ainda segundo a autora, um dos motivos para esse aumento, além da introdução de novas tecnologias, é a assimetria de informação, tanto de produtos quanto de preços, principalmente em relação aos materiais especiais ${ }^{29}$.

De acordo com a Tabela 4, quando se compara o aumento dos gastos entre os beneficiários mais velhos e os mais novos, observa-se que com materiais foi ligeiramente maior para os primeiros (148\% versus 143\%), assim como para medicamentos (66\% versus $52 \%)$. A população mais velha ainda sofreu mais com o aumento dos gastos com terapias (33\% versus 9\%). Na comparação entre sexos, observam-se aumentos superiores nos custos do sexo masculino em todas as categorias, com destaque também para o aumento nos gastos com materiais (179\% versus $120 \%$ ) e medicamentos (77\% versus $60 \%$ ), os maiores dispêndios de internação.

\section{Considerações finais e conclusão}

Tendo em vista a relevância da discussão sobre o aumento dos custos das operadoras de saúde no Brasil, em função do envelhecimento da sua

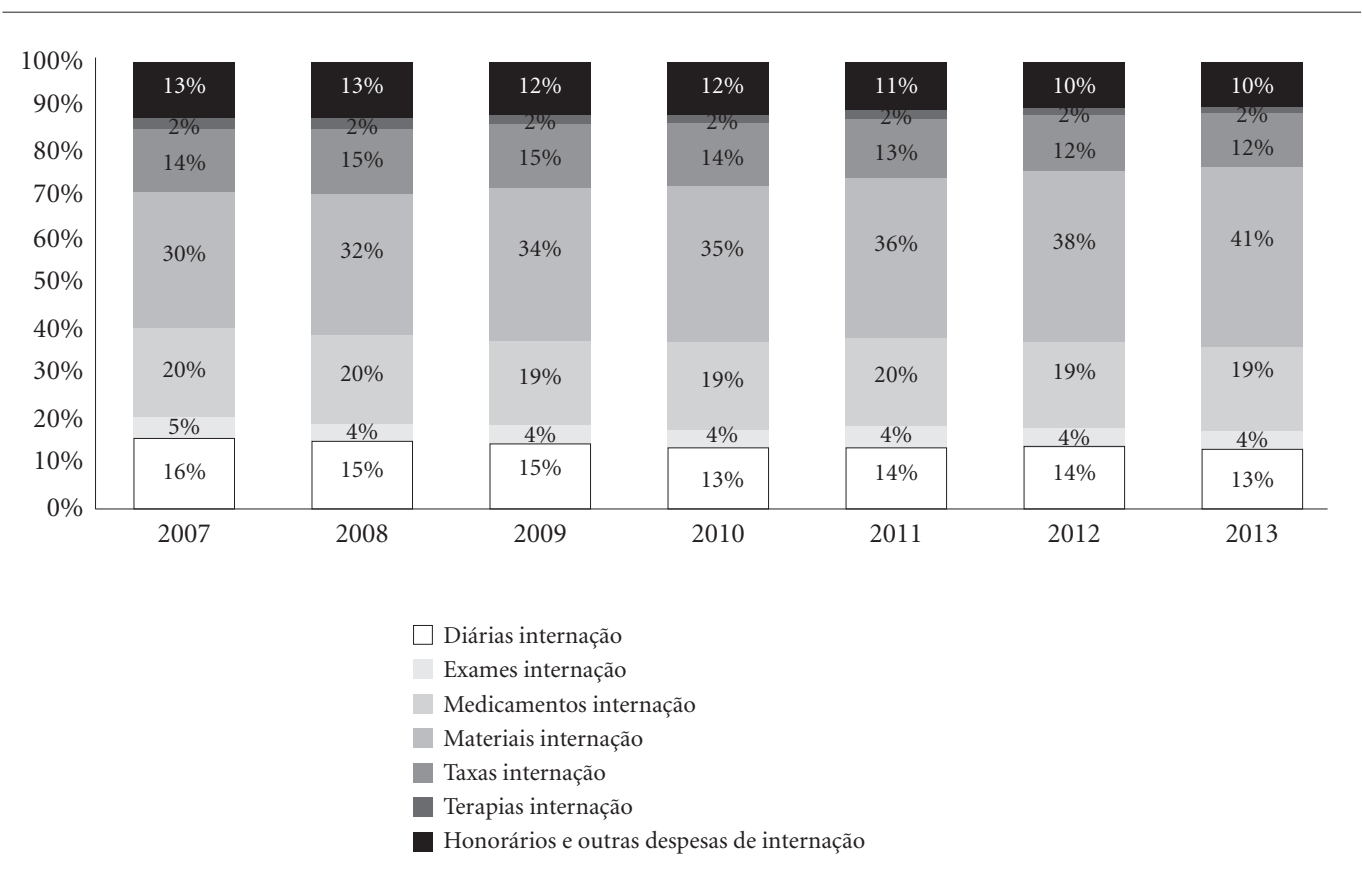

Gráfico 3. Evolução da representatividade dos gastos com internação.

Tabela 4. Aumento dos gastos de internação de 2007 a 2013 na Operadora de Saúde.

\begin{tabular}{lccccc}
\hline $\begin{array}{c}\text { Gastos com Internação } \\
\text { (por diária de internação) }\end{array}$ & Geral & $<$ de $\mathbf{6 0}$ anos & $>$ de $\mathbf{6 0}$ anos & Feminino & Masculino \\
\hline Diária Hospitalar & $52 \%$ & $48 \%$ & $48 \%$ & $48 \%$ & $56 \%$ \\
Exames & $63 \%$ & $77 \%$ & $51 \%$ & $49 \%$ & $81 \%$ \\
Medicamentos & $67 \%$ & $52 \%$ & $66 \%$ & $60 \%$ & $77 \%$ \\
Materiais & $146 \%$ & $143 \%$ & $148 \%$ & $120 \%$ & $179 \%$ \\
Taxas & $52 \%$ & $68 \%$ & $45 \%$ & $48 \%$ & $57 \%$ \\
Terapias & $24 \%$ & $9 \%$ & $33 \%$ & $19 \%$ & $34 \%$ \\
Honorários e Outros & $45 \%$ & $44 \%$ & $54 \%$ & $28 \%$ & $67 \%$ \\
\hline
\end{tabular}


população, este trabalho teve como objetivo contribuir com a exposição de como se deu a evolução da utilização e dos gastos, no período recente de 2007 a 2013, de uma operadora, cujo rápido envelhecimento dos beneficiários a torna única. Com 36\% dos beneficiários acima dos 60 anos de idade em 2013, a operadora de saúde objeto deste estudo apresenta um perfil populacional próximo ao esperado somente para o ano de $2050^{23}$ nos planos de saúde do país.

As principais conclusões com relação ao grau de utilização mostram que a taxa de uso do plano aumentou consideravelmente, em especial para a população mais velha que vem empregando mais o plano em 2013 do que em 2007. Os homens mais velhos estão mais preocupados com a saúde e passaram a utilizar os serviços da operadora no mesmo nível das mulheres de mesma faixa etária. Isto mostra uma mudança no comportamento masculino em relação à sua saúde, fato que merece atenção das operadoras. Aproveitar o maior grau de utilização por parte dos homens pode ser uma oportunidade para trabalhos de caráter preventivo, portanto, entender melhor este fenômeno poderá trazer benefícios para a redução dos gastos das operadoras de saúde com a população mais velha.

Por sua vez, é interessante perceber que enquanto a taxa de internação das mulheres acima de 60 anos teve uma redução de 8 pontos percentuais, para os homens da mesma faixa etária houve um aumento de quase 10 pontos percentuais, representando mais um fator inflacionário para as operadoras de saúde. Ainda com relação às internações, o estudo detectou que o tempo médio de permanência nos hospitais subiu para ambas as faixas etárias estudadas. Isto leva a pensar que houve um agravamento nos casos de internação, porém, novas pesquisas poderiam investigar o aumento no tempo de internação dos pacientes.

Os gastos com internação são preocupantes, pois continuam sendo os mais expressivos e apresentando forte tendência de subida para os próximos anos. Os gastos por diária de internação tiveram um aumento médio muito acima da inflação do período, em todas as faixas etárias, corroborando a literatura da área ${ }^{10,25}$. Dentro das várias categorias de custos com internação, merecem destaque os gastos com materiais e medicamentos.

Isto mostra que existem alguns direcionadores de custos que gestores públicos e privados deveriam olhar com maior atenção para tentar frear os gastos da saúde. O crescimento dos gastos de uma operadora de saúde não pode ser explicado somente pelo envelhecimento de seus benefici- ários. Embora represente um vetor importante, pôde-se verificar que os gastos estão acima da inflação em todas as categorias e nas mais diversas faixas etárias.

Outros estudos poderiam ser realizados para entender, de maneira mais profunda, o aumento expressivo dos gastos com materiais e medicamentos. Pesquisas sobre assimetria de informação e adoção de novas tecnologias poderiam ajudar a explicar estes gastos.

Durante a realização da pesquisa pôde-se perceber a dificuldade que as operadoras têm em obter informações precisas e completas de seus beneficiários e de suas enfermidades, bem como dos prestadores de serviços e de toda a cadeia de valor da saúde ${ }^{16}$. Certamente um trabalho de integração e compartilhamento de informação em toda a cadeia poderá trazer benefícios enormes para todos os players da saúde, sejam públicos ou privados.

Cabe ainda registar a grande limitação deste estudo. $\mathrm{O}$ fato de ter-se utilizado uma única operadora de plano de saúde pode trazer certo viés na generalização das conclusões de pesquisa, mas por outro lado permitiu um mergulho mais profundo no entendimento dos direcionadores de custo e na utilização da operadora por parte de seus beneficiários.

Por fim, este artigo buscou contribuir para o entendimento da evolução dos custos de uma operadora de saúde que sofre com o envelhecimento de seus beneficiários. Espera-se que este estudo possa ajudar outras operadoras tanto na comparação de seus dados, como uma fonte de benchmarking, quanto no processo de tomada de decisão sobre como gerenciar os seus principais gastos de forma inteligente e sustentável.

\section{Colaboradores}

ALCM Duarte trabalhou na análise e conclusão dos dados; FM Oliveira na análise dos dados; AA Santos na contextualização e referencial bibliográfico; BFC Santos na análise e conclusão dos dados. 


\section{Referências}

1. Montekio VB, Medina G, Aquino R. The health system of Brazil. Salud Publica Mex 2011; 53(2):s120-131.

2. Paim J, Travassos C, Almeida C, Bahia L, MacInko J. The Brazilian health system: History, advances, and challenges. Lancet [Internet]. 2011 May 21 [cited 2014 Mar 20];377(9779):1778-97. Available from: http:// www.ncbi.nlm.nih.gov/pubmed/21561655

3. CONASS. Para entender a gestão do SUS. In: SUS, editor. Coleção Progestores [Internet]. 20a ed. Brasília: Conselho Nacional de Secretários de Saúde; 2007. p. 234. [cited 2014 May 5]. Available from: http://extranet.saude. prefeitura.sp.gov.br/biblioteca/livros/colecao-progestores-2013-para-entender-a-gestao-do-sus/livro11.pdf

4. Portal Brasil. Saúde suplementar deve fechar 2013 com 50 milhões de beneficiários [Internet]. Portal Brasil. 2014 [cited 2014 May 5]. p. 2013-5. Available from: http://www.brasil.gov.br/saude/2013/12/saude-suplementar-deve-fechar-2013-com-50-milhoes-de-beneficiarios

5. Agência Nacional de Saúde Suplementar. Caderno de Informacão da Saúde Suplementar: beneficiários, operadoras e planos [Internet]. Rio de Janeiro; 2015 Mar. [cited 2015 Sep 14]. Available from: http://www.ans. gov.br/portal/site/informacoesss/informacoesss.asp

6. Agência Nacional de Saúde Suplementar. Dados e indicadores do setor [Internet]. 2015 [cited 2015 Sep 14]. Available from: http://www.ans.gov.br/perfil-do-setor/ dados-e-indicadores-do-setor

7. Leal RM, Matos JBB. Planos de saúde: uma análise dos custos assistenciais e seus componentes. Rev Adm Empres 2009; 49(4):447-458.

8. Nunes A. O envelhecimento populacional e as despesas do Sistema Único de Saúde. In: Camarano AA, organizadora. Os novos idosos Bras muito além dos 60 ? Rio de Janeiro: IPEA; 1999. p. 427-449.

9. Reis A, Mansini G, Leite F. Atualização das projeções de gastos com saúde divulgados no relatório "Envelhecimento populacional e os desafios para o sistema de saúde brasileiro". Instituto de Estudos De Saúde Suplementar 2013; 1-12.

10. Santos GMM, Maria G. Materiais e Medicamentos: vetores de custo no setor de saúde suplementar. São Paulo: IESS; 2013.

11. Reis ASA. A importância de Materiais e Medicamentos nos gastos médicos: dados de uma operadora no período de 2007 a 2012. São Paulo: IESS; 2014.

12. Fundação Instituto de Pesquisas Econômicas. Índice de Preços ao Consumidor [Internet]. 2015 [cited 2015 Jan 30]. Available from: http://www.fipe.org.br/pt-br/indices/ipc/\#indice-mensal\&macum

13. Zucchi P, Del Nero C, Malik AMA. Gastos em saúde: os fatores que agem na demanda e na oferta dos serviços de saúde. Saúde e Soc [Internet]. 2000 [cited 2014 Apr 23]; 9(1-2):127-50. Available from: http://www.scielo.br/scielo.php?script=sci_arttext\&pid=S010412902000000100010\&lng=en\&nrm=iso

14. Kanamura AH, Viana ALD. Gastos elevados em plano privado de saúde: com quem e em quê. Rev Saude Publica. 2007;41(5):814-820.

15. Instituto de Estudos de Saúde Suplementar. Guia da Saúde Suplementar iess. $2^{\text {nd }}$ ed. São Paulo; 2013;44. [cited 2015 Jan 11]. Available from: http://www.scribd. com/doc/219670881/phpHXvZFJ
16. Dye C, Reeder JC, Terry RF. Research for universal health coverage. Sci Transl Med 2013; 5(199):199ed13.

17. Lima CRM. A avaliação do custo eficácia das intervenções em organizações de saúde. Rev Adm Empres 1998; 38(2):62-73.

18. Freitas RM, Cherchiglia ML, Lana FCF, Malta DC, Andrade EIG. Estudos dos modelos assistenciais praticados por operadoras de planos privados de saúde. Physis 2011; 21(4):1561-1577.

19. Piva LC, Baptista PP. Relação entre a adoção de práticas de promoção da saúde e prevenção de doenças e a percepção de valor para clientes de plano de saúde [Internet]. $33^{\text {rd }}$ ed. XXXIII Encontro da Anpad. [São Paulo, Brasil]: ANPAD; 2008. [cited 2015 Jan 11]. Available from: http://www.anpad.org.br/admin/pdf/MKT2146. pdf

20. Carneiro LAF, Campino ACC, Leite F, Rodrigues CG, Santos GMM, Silva ARA. Envelhecimento populacional e os desafios para o sistema de saúde brasileiro [Internet]. São Paulo; 2013. [cited 2015 Jan 11]. Available from: http://www.iess.org.br/html/1apresentao.pdf

21. Instituto Brasileiro de Geografia e Estatística (IBGE). Sintese de Indicadores - 2013. Pesquisa Nacional por Amostra de Domicílios. Rio de Janeiro: IBGE; 2014.

22. Agência Nacional de Saúde Suplementar. Plano de cuidado para idosos na saúde suplementar [Internet]. Rio de Janeiro; 2012. [cited 2015 Jan 11]. Available from: http://bvsms.saude.gov.br/bvs/publicacoes/plano_cuidado_idosos.pdf

23. Andrade MV, Maia AC. Demanda por planos de saúde no Brasil. 2006;2:1-21. [Internet]. [cited 2015 Jan 11]. Available from: http://www.anpec.org.br/encontro2006/artigos/A06A106.pdf

24. Andrade MV, Maiab AC, Rodrigues CG. Indicadores de gastos com serviços médicos no setor de saúde suplementar no Brasil: o caso Sabesprev. In: Belo Horizonte; 2010 [cited 2014 May 30]. p. 1-22. Available from: http://150.164.82.140/pesquisas/td/TD 403.pdf

25. Newhouse JP. Medical Care Costs: How Much Welfare Loss? J Econ Perspect 1992; 6(3):3-21.

26. Ferreira DQG. Os determinantes da integração vertical na saúde suplementar segundo a teoria dos custos de transação [tese]. Rio de Janeiro: UFRJ; 2013.

27. Sampieri RH, Collado CF, Lucio MPB. Metodologia de pesquisa. $5^{\text {a }}$ ed. Porto Alegre: AMGH; 2013.

28. Carlos GA. Como elaborar projetos de pesquisa. São Paulo: Atlas; 2002.

29. Leite F. Por que os custos com internação dos planos de saúde são os que mais crescem? IESS 2013;1-9. [cited 2014 Apr 23]. Available from: http://www.iess.org.br/ html/TDIESS0048pqcustoscrescem.pdf

30. Andrade MV, Maia AC, Rodrigues CG. Indicadores de gastos com serviços médicos no setor de saúde suplementar no Brasil. Rev Bras Estud Popul [Internet]. Rio de Janeiro; 2013. [cited 2014 Apr 23];30:103-118.Available from: http://www.rebep.org.br/index.php/revista/ article/view/386

Artigo apresentado em 13/10/2015

Aprovado em 21/03/2016

Versão final apresentada em 23/03/2016 